\title{
Silicon Detectors for the SLHC \\ - an Overview of Recent RD50 Results
}

\author{
Giulio Pellegrini ${ }^{1}$ \\ Centro Nacional de Microelectronica CNM-IMB-CSIC, \\ Barcelona Spain \\ E-mail: giulio.pellegrinieimb-cnm.csic.es
}

\section{On behalf of CERN RD50 collaboration}

\begin{abstract}
It is foreseen to significantly increase the luminosity of the Large Hadron Collider(LHC) at CERN around 2018 by upgrading the LHC towards the sLHC (Super-LHC). Due to the radiation damage to the silicon detectors used, the physics experiment will require new tracking detectors for sLHC operation. All-silicon central trackers are being studied in ATLAS, CMS and $\mathrm{LHCb}$, with extremely radiation hard silicon sensors on the innermost layers. The radiation hardness of these new sensors must surpass the one of LHC detectors by roughly an order of magnitude. Within the CERN RD50 collaboration, a massive R\&D programme is underway to develop silicon sensors with sufficient radiation tolerance. Among the R\&D topics are the development of new sensor types like 3D silicon detectors designed for the extreme radiation levels of the sLHC. We will report on the recent results obtained by RD50 from tests of several detector technologies and silicon materials at radiation levels corresponding to SLHC fluences. Based on these results, we will give recommendations for the silicon detectors to be used at the different radii of SLHC tracking systems.
\end{abstract}

European Physical Society Europhysics Conference on High Energy Physics EPS-HEP 2009,

Krakow , Poland

July 16-22, 2009

\footnotetext{
1 Speaker
} 


\section{Introduction}

Current estimates on the radiation environment of the tracker region at SLHC rely on simulations for the LHC experiments. Expected fluences can be scaled by integrated luminosity, while energy spectra and particle composition are assumed to not change since the collision energy will stay at $14 \mathrm{TeV}$ and the surrounding detectors will only be slightly modified. One expects a maximum total fluence of $2.5 \times 10^{16} \mathrm{n}_{\mathrm{eq}} \mathrm{cm}^{-2}$ for the pixel region $(<20 \mathrm{~cm})$ and a minimum of $4 \times 10^{14} \mathrm{~cm}^{-2}$ for the outer strip region. To cope with the expected high density of particle tracks one has to use a silicon tracker with finer segmentation compared to the current device. For example, in an intermediate region $(20 \mathrm{~cm}<\mathrm{R}<60 \mathrm{~cm})$ short strips of $2.5 \mathrm{~cm}$ or 5 $\mathrm{cm}$ length might be appropriate. It is also important to realize that the ratio of neutron to charged hadron fluence changes over the radius. This is of increasing importance since NIEL ${ }^{1}$ violating damage processes have been found [1]. The radiation in the innermost region is dominated by charged hadrons while radiation from $40 \mathrm{~cm}$ onwards is dominated by neutrons.

\subsection{The RD50 Collaboration}

CERN has initiated research programs to and possible solutions for tracking detectors at the HEP experiments. The RD50 project - "Radiation hard semiconductor devices for very high luminosity colliders" [2], covers many possible semiconductor materials and technologies, and consists of 47 institutes with 248 members The approach is to understand the relation between microscopic defects and macroscopic properties and to use this knowledge to engineer new materials with higher radiation hardness. In parallel new detector technologies are being explored and finally the improvements are applied to sensor prototypes for collider experiments.

RD50 collaboration has five research lines which are: Defect and Material Characterization, Defect Engineering, Pad Detector Characterization, New Structures and Full Detector Systems.

\subsection{Recent Results}

Recent studies within the defect and material characterization research line have identified three hole trap levels $\mathrm{H}(116 \mathrm{~K}), \mathrm{H}(140 \mathrm{~K})$ and $\mathrm{H}(152 \mathrm{~K})$, which had been proven to be responsible for the long term annealing effects in silicon [3]. The levels are in the lower half of the band gap and act as acceptors, i.e. they contribute with negative space charge to the effective doping concentration $\left(\mathrm{N}_{\text {eff }}\right)$ of depleted sensors. The concentration as measured by TSC increases slowly with annealing time in correlation with Neff as measured by CV. Combining these findings with the annealing behaviour of the previously found bistable donors [4], one can add up the effective space charges obtained from the measured defect concentrations and compare this with $\mathrm{N}_{\text {eff }}$ values extracted from $\mathrm{CV}$ measurements. These comparisons show an excellent agreement, which is an outstanding progress in the understanding of the annealing behaviour of the full depletion voltage.

\footnotetext{
${ }^{1}$ Non Ionizing Energy Loss
} 
One interesting result of the defect engineering research line is the transformation of $\mathrm{p}$ type magnetic Czochralski ( $\mathrm{MCz}$ ) silicon into effective n-type by introducing thermal donors with heat treatment at $450{ }^{\circ} \mathrm{C}$ [5]. This is a cost effective method to control the full depletion voltage of highly oxygenated material. The final heat treatment after processing $\mathrm{n}$-type $\mathrm{MCz}$ silicon produces thermal donors as well, thus reducing the resistivity and increasing the full depletion voltage. Therefore $\mathrm{n}$-type $\mathrm{MCz}$ with low full depletion voltage need special care during the final process steps or the use of a p-type substrate.

New results in the characterization of pad detectors have shown that already at moderate fluences and low voltages the CCE values in the saturation region are higher than expected and cannot be explained by the standard trapping model, which might need to be extended. Moreover, the CCE in highly irradiated thin epitaxial diodes shows a strong increase at high bias voltages, sometimes clearly exceeding the value of non-irradiated samples (Fig. 1). This can only be explained by charge multiplication due to very high electric fields in thin diodes after strong irradiation.

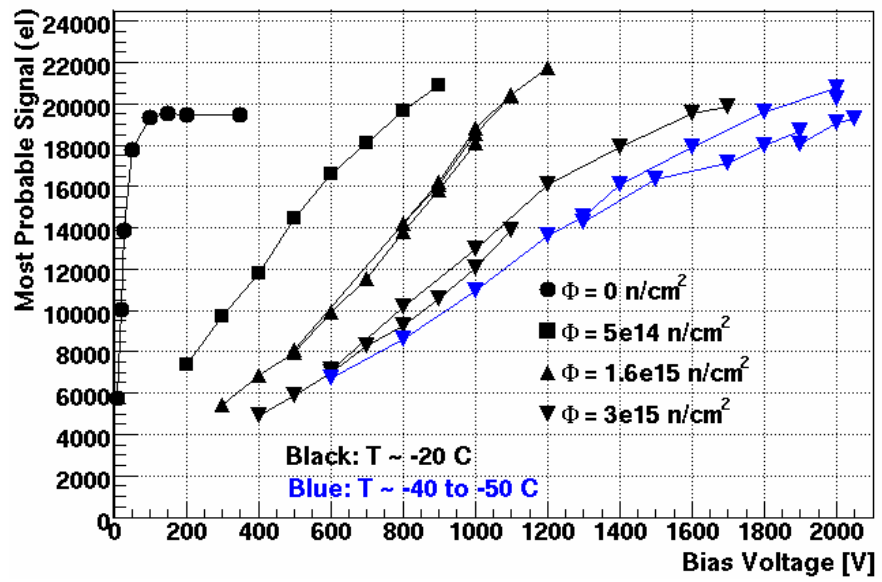

Figure 1. Charge multiplication at high bias voltages [9].

Most of the work of the new structure group has been focused in the development of 3D detectors [6]. At very high fluences the CCE is limited by trapping effects, i.e. one has to work with small collection distances. To make use of a thick volume in which electron-hole pairs are generated and to collect the charge carriers after a short drift distance, 3D sensors have been developed. 3D detectors provide electrodes in form of pillars reaching ideally through the complete bulk.. The drift distance for the charge carriers in these sensors is defined by the pitch of the pillars and not the sensor thickness. 3D sensor producers in our collaboration are $\mathrm{CNM}^{2}$ and $\mathrm{FBK}^{3}$. Both $\mathrm{p}$ - and n-type devices have been produced and tested successfully $[7,8]$.

The full detector system group has largely tested that p-type FZ sensors appear to perform well after strong irradiation. At a fluence of $1 \times 10^{16} \mathrm{n}_{\mathrm{eq}} \mathrm{cm}^{-2}$ they still show signals of 6500 electrons in $300 \mu \mathrm{m}$ thick strips sensors biased at $900 \mathrm{~V}$ (Fig. 2). These sensors also show the surprising feature that the charge collection does not change with time. Even after 400 minutes at $80^{\circ} \mathrm{C}$ no change could be measured [11].

\footnotetext{
${ }^{2}$ Centro Nacional de Microelectronica (IMB-CNM, CSIC), Barcelona, Spain

${ }^{3}$ Fondazione Bruno Kessler, Povo, Trento, Italy
} 


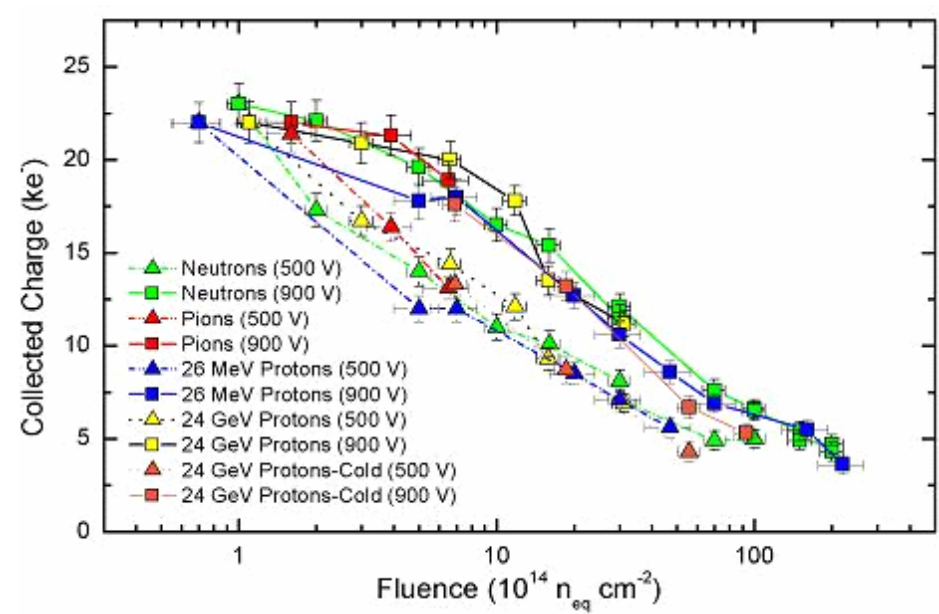

Figure 2: Collected charge for n-in-p FZ silicon strip sensors. [10]

\section{Recommendations}

The main task of RD50 is to find a suitable semiconductor technology for a central vertex detector and tracker at SLHC. Summarizing the results of the past years one can conclude on the following recommendations:

The outer regions starting at a radius of $20 \mathrm{~cm}$ will be exposed to a total fluence of less than $1 \times 10^{15} \mathrm{n}_{\mathrm{eq}} \mathrm{cm}^{-2}$. For this region $300 \mu \mathrm{m}$ p-type FZ silicon can be used with a collected charge of more than 10000 electrons at $500 \mathrm{~V}$. In addition, the charge collection is not a affected by annealing effects. Using $n$-in-p instead of $n$-in-n sensors allows to have a single sided process, which makes the production cheaper.

Sensors in the inner region (from $4 \mathrm{~cm}$ to $20 \mathrm{~cm}$ ) will be exposed to a total fluence of up to $2.5 \times 10^{16} \mathrm{n}_{\mathrm{eq}} \mathrm{cm}^{-2}$, mainly produced by charged hadrons. When using planar sensors in this region one has to apply bias voltages as high as possible. Thin sensors would allow lower bias voltage, but not necessarily less leakage current. The 3D sensors are designed to cope with this trapping problem. They collect the charge carriers within short distances while the entire thickness is available for electron-hole pair creation. The technology has still to be optimized to allow a cost effective production.

In conclusion, technologies and materials for a silicon tracker at SLHC are available. We will further investigate which solution would be the optimum for the operation of the experiment.

\section{References}

[1] M. Huhtinen, Nucl. Instr. and Meth. A 491 (2002), p. 194

[2] http://www.cern.ch/rd50

[3] I. Pintilie et al., APL 92 (2008), 024101.

[4] I. Pintilie et al., Nucl. Instr. and Meth. A 552 (2005), p. 56 
[5] J. Härkönen et al., Nucl. Instr. and Meth. A 552 (2005), p. 43

[6] "S.I. Parker et al., Nucl. Instr. and Meth. A 395 (1997), p. 328

[7] C. Fleta et al., Nucl. Instr. and Meth. A 607, (2009), p. 89

[8] A. Zoboli et al., Nucl. Instr. and Meth. A 604 (2009), p. 238

[9] I. Mandic et al., Nucl. Instr. and Meth. A 603 (2009) 263-267.

[10] A. Affolder et.al., "Charge collection efficiencies of planar silicon detectors after reactor neutron, pion, and proton doses up to $2 \mathrm{e} 16 \mathrm{neq} / \mathrm{cm} 2 "$, presented at the 1 st international conference on Technology and Instrumentation in Particle Physics (TIPP09), Tsukuba, Japan, March 11-17th, 2009 and accepted for publication in NIMA.

[11] G. Casse et al., Nucl. Instr. and Meth. A 568 (2006), p. 46 